\title{
Influence of Birth Weight on the Apo E Genetic Determinants of Plasma Lipid Levels in Children
}

\author{
CARMEN GARCÉS, MERCEDES BENAVENTE, HENAR ORTEGA, RAFAEL RUBIO, \\ MIGUEL ANGEL LASUNCIÓN, FERNANDO RODRÍGUEZ ARTALEJO, \\ JACINTO FERNÁNDEZ PARDO, AND MANUEL DE OYA
}

Unidad de Lipidos, Fundación Jiménez Díaz, Universidad Autónoma de Madrid, Avda. Reyes Católicos, 2 28040 Madrid, Spain [C.G., M.B., R.R., M.d.O.], Servicio de Bioquímica-Investigación. Hospital Ramón y Cajal, Universidad de Alcalá, Madrid, Carretera de Comenar km. 928034 Madrid, Spain [H.O., M.A.L.],

Departamento de Medicina Preventiva y Salud Pública, Universidad Autónoma de Madrid, Spain, Arzobispo Morcillo s/n 28029 Madrid, Spain [F.R.A.], Hospital General Universitario, Avda. I. Jorge Palacios 130003 Murcia, Spain [J.F.P.]

\begin{abstract}
To evaluate the influence of birth weight on apolipoprotein (apo) E genetic determinants of plasma lipids levels in prepubertal children we studied 933 healthy children (491 males and 442 females) 6 to 8 years old (mean age of $6.7 \mathrm{y}$ ), whose weight was recorded at birth. Plasma lipid and apolipoprotein concentrations and apo E genotypes were determined. We observed a greater effect of the apo E polymorphism on total cholesterol (TC), LDL-cholesterol (LDL-C) and especially apo B levels in children with birth weight in the lower tertile compared with those with birth weights in higher tertiles. Taking the $\epsilon 3$ allele homozygosity as reference, in boys with birth weights in the low tertile the overall lowering effect of the $\epsilon 2$ allele on TC, LDL-C and apo B was greater $(10.5 \%(p<0.01), 20.2 \%(p<0.01)$ and $18.8 \%(p<0.01)$, respectively) than in those in the highest tertile (5.6\% on TC, $10.3 \%$ on LDL-C and $12.6 \%(p<0.01)$ on apo B). A similar trend in this effect between tertiles of birth weight was also observed in girls. For both sexes, linear regression analysis
\end{abstract}

\section{ABSTRACT}

demonstrates a positive and significant interaction between birth weight and $\epsilon 2$, which may explain the fact that the decrease in TC, LDL-C and apo B associated with the $\epsilon 2$ allele is more marked the lower the birth weight. Taking into account the prevalence of apo E polymorphism, and that appears to be the main genetic factor affecting plasma lipids, the interaction of apo E genotype and birth weight could be an important determinant of TC, LDL-C and apo B levels, and, as a consequence, of atherosclerosis. (Pediatr Res 52: 873-878, 2002)
apo, apolipoprotein
TC, total cholesterol
TG, triglycerides
HDL-C, HDL-cholesterol
LDL-C, LDL-cholesterol
CHD, coronary heart disease

Coronary heart disease (CHD) and its risk factors, including abnormalities in lipid metabolism, are associated with small size at birth and low weight during infancy $(1,2)$. Genetic composition also determines differences in plasma lipid levels and susceptibility to atherosclerosis. Although the risk of CHD due to both environmental and genetic influences is well known, the extent of interactions between these factors has not been well characterized.

Apolipoprotein E (apo E) is one of the key regulators of plasma lipid levels by affecting the hepatic binding, uptake, and catabolism of several classes of lipoproteins (3). Apo E

Received April 3, 2001; accepted July 3, 2002.

Correspondence: Prof. Manuel de Oya, Unidad de Lípidos, Fundación Jiménez Díaz, Avda. Reyes Católicos, 2, 28040 Madrid, Spain; e-mail: moya@fjd.es

This study was supported by grants from Comunidad Autónoma de Madrid (08.4/0006/ 1997) and Fundación Ramón Areces.

DOI: 10.1203/01.PDR.0000037140.57784.8B genotypes are one of the most important genetic determinants of atherogenesis. Apo E appears in three isoforms E2, E3, and E4, encoded by the corresponding alleles $\epsilon 2, \epsilon 3$, and $\epsilon 4$. Previous studies have documented the impact of these mutations on CHD (4-6) and the effects of these alleles on the normal variation of lipid levels in adult populations. Heterozygous subjects for the $\epsilon 2$ and $\epsilon 4$ alleles had, respectively, lower and higher total cholesterol (TC) and LDL-C levels than subjects carrying the $\epsilon 3 \epsilon 3$ genotype (7-9). This influence of the apo E polymorphisms on the metabolism of the apo B-containing lipoproteins appears to be present in the 1 st years of life $(10-13)$. However, the influence of in utero growth and metabolism on the apo E genetic contributions to plasma lipid levels remains unclear. Thus, in the present work, we analyzed the influence of birth weight on the apo E genotype determinants of plasma lipids levels on later steps of life by studying the apo E effect on lipid 
variables in a large, population-based sample of prepubertal children in Spain.

\section{SUBJECTS AND METHODS}

Population. The cohort population included 933 healthy school children (491 males and 442 females) 6 to 8 y old (mean age of $6.7 \mathrm{y}$ ) who participated in a voluntary survey of cardiovascular risk factors in Spain (14). Subjects accepted for study were required to submit birth weight as recorded in a legal birth certificate. All were free of any endocrine, metabolic, hepatic,

Table 1. Anthropometric variables, plasma lipids, and apolipoprotein levels (mean (SD)), by gender

\begin{tabular}{lccc}
\hline & Boys $(n=491)$ & Girls $(n=442)$ & $p$-Values* \\
\hline Age $(\mathrm{yr})$ & $6.69(0.66)$ & $6.70(0.66)$ & - \\
Weight $(\mathrm{kg})$ & $26.8(5.30)$ & $26.6(5.43)$ & - \\
Height $(\mathrm{m})$ & $1.26(0.06)$ & $1.25(0.07)$ & - \\
BMI $\left(\mathrm{kg} / \mathrm{m}^{2}\right)$ & $16.9(2.4)$ & $17.0(2.5)$ & - \\
Birth weight $(\mathrm{kg})$ & $3.42(0.54)$ & $3.23(0.52)$ & $\dagger$ \\
TC $(\mathrm{mg} / \mathrm{dl})$ & $182.3(28.7)$ & $183.4(28.8)$ & - \\
HDL-C $(\mathrm{mg} / \mathrm{dl})$ & $59.7(13.5)$ & $58.2(13.8)$ & - \\
TG $(\mathrm{mg} / \mathrm{dl})$ & $71.4(25.4)$ & $74.3(27.1)$ & - \\
LDL-C $(\mathrm{mg} / \mathrm{dl})$ & $108.2(27.8)$ & $110.2(27.1)$ & - \\
Apo A-I (mg/dl) & $138.4(19.0)$ & $135.5(19.10)$ & $\ddagger$ \\
Apo B $(\mathrm{mg} / \mathrm{dl})$ & $69.1(15.0)$ & $71.3(15.0)$ & $\ddagger$ \\
\hline
\end{tabular}

* $t$ Student.

$\dagger p<0.01$.

$\ddagger p<0.05$. or renal disorder. The study protocol complied with Helsinki Declaration guidelines and Spanish legal provisions governing clinical research on humans, and was approved by the Clinical Research Ethics Committee of the Fundación Jiménez Díaz in Madrid. Parents were required to sign a written consent for participation of their children in the study. Sampling was randomized and stratified by pools of school centers in each participating city.

Anthropometric measurements. Height and weight were determined when children were lightly dressed and without shoes. Height was measured to the nearest $0.1 \mathrm{~cm}$ using a portable stadiometer and weight was recorded to the nearest $0.1 \mathrm{~kg}$ using a standardized electronic digital scale. From these measurements a body mass index (BMI, weight in kilograms divided by the square of height in meters, $\mathrm{kg} / \mathrm{m}^{2}$ ) was calculated. A questionnaire was provided along with consent forms and parents were asked to provide information regarding the child's general health and about family history of dyslipidemia or CHD, and to report birth weight from a birth certificate.

Blood sampling, lipid and apolipoprotein analyses, and DNA extraction. Fasting (12-hours) venous blood samples were obtained from every child early in the morning by venipuncture. Plasma cholesterol and triglyceride levels were determined enzymatically (Menarini Diagnostics) with a RA1000 Autoanalyzer. The coefficients of variation of the methods were $2.06 \%$ for cholesterol determinations and $3.42 \%$ for triglycerides determinations. HDL-cholesterol (HDL-C) was

Table 2. Plasma lipid and apolipoprotein levels (mean (SD)), by birth weight tertiles and apo E genotype, in boys

\begin{tabular}{|c|c|c|c|c|}
\hline & $\epsilon 2 \epsilon 3^{*} n=48$ & $\epsilon 3 \epsilon 3 n=328$ & $\epsilon 3 \epsilon 4 * n=87$ & All $n=463$ \\
\hline \multicolumn{5}{|l|}{$\mathrm{TC}(\mathrm{mg} / \mathrm{dl})$} \\
\hline Low tertile & $163.4(27.8) \dagger$ & $182.5(23.1)$ & $190.7(29.6)$ & $182.3(27.0)$ \\
\hline Medium tertile & $171.3(23.1)$ & $181.1(21.8)$ & $183.9(25.2)$ & $183.8(24.2)$ \\
\hline High tertile & $170.6(23.5)$ & $180.7(23.4)$ & $183.8(26.1)$ & $180.6(24.3)$ \\
\hline Whole group & $168.9(24.8) \dagger$ & $182.8(23.4)$ & $186.8(26.7)$ & $182.2(25.2)$ \\
\hline \multicolumn{5}{|l|}{ LDL-C (mg/dl) } \\
\hline Low tertile & $86.2(26.9) \dagger$ & $108.0(23.5)$ & $116.9(28.9)$ & $107.8(27.0)$ \\
\hline Medium tertile & $90.3(19.0) \dagger$ & $107.3(21.3)$ & $112.8(24.9)$ & $110.0(24.2)$ \\
\hline High tertile & $95.1(21.3)$ & $106.1(21.3)$ & $113.5(24.6)$ & $106.7(22.7)$ \\
\hline Whole group & $90.9(22.6) \dagger$ & $108.7(22.8)$ & $115.4(25.6)$ & $108.1(24.8)$ \\
\hline \multicolumn{5}{|l|}{ apo B (mg/dl) } \\
\hline Low tertile & $55.4(12.2) \dagger$ & $68.2(12.5)$ & $74.5(19.2)$ & $68.3(15.1)$ \\
\hline Medium tertile & $54.7(10.0) \dagger$ & $68.0(12.8)$ & $71.0(11.1)$ & $68.4(13.7)$ \\
\hline High tertile & $59.2(10.6) \dagger$ & $67.7(10.7)$ & $74.0(13.7) \ddagger$ & $68.3(12.1)$ \\
\hline Whole group & $56.6(11.0) \dagger$ & $68.7(12.4)$ & $73.3(14.5) \dagger$ & $68.3(13.7)$ \\
\hline \multicolumn{5}{|l|}{$\mathrm{TG}(\mathrm{mg} / \mathrm{dl})$} \\
\hline Low tertile & $70.7(18.2)$ & $72.0(23.4)$ & $72.2(24.0)$ & $72.7(24.0)$ \\
\hline Medium tertile & $70.9(18.1)$ & $72.2(24.7)$ & $77.5(25.3)$ & $72.9(23.4)$ \\
\hline High tertile & $73.4(16.8)$ & $70.1(22.2)$ & $76.4(23.7)$ & $71.8(22.0)$ \\
\hline Whole group & $72.1(17.6)$ & $71.5(23.1)$ & $74.8(24.3)$ & $72.5(23.1)$ \\
\hline \multicolumn{5}{|l|}{ HDL-C (mg/dl) } \\
\hline Low tertile & $62.8(13.2)$ & $59.7(14.4)$ & $58.9(11.7)$ & $59.7(13.8)$ \\
\hline Medium tertile & $59.6(8.6)$ & $57.0(11.2)$ & $54.8(11.6)$ & $58.9(13.4)$ \\
\hline High tertile & $60.3(16.9)$ & $60.2(13.8)$ & $54.9(12.2)$ & $59.1(13.9)$ \\
\hline Whole group & $63.2(14.9)$ & $59.4(13.7)$ & $56.1(11.9) \ddagger$ & $59.5(13.7)$ \\
\hline \multicolumn{5}{|l|}{ apo A-I (mg/dl) } \\
\hline Low tertile & $138.1(19.1)$ & $138.5(15.8)$ & $134.0(19.5)$ & $137.5(16.9)$ \\
\hline Medium tertile & $144.0(20.8)$ & $139.0(18.1)$ & $134.5(18.1)$ & $141.6(21.2)$ \\
\hline High tertile & $139.8(17.9)$ & $139.5(18.4)$ & $132.2(21.8)$ & $138.1(19.1)$ \\
\hline Whole group & 144.9 (23.9) & $139.3(17.4)$ & $134.4(20.5) t$ & $139.0(19.1)$ \\
\hline
\end{tabular}

* Tukey test: $p$-values for differences in lipid levels between the $\varepsilon 3 \varepsilon 2$ or $\varepsilon 3 \varepsilon 4$ genotypes respectively, and the $\varepsilon 3 \varepsilon 3$ genotype.

$\dagger p<0.01$.

$\ddagger p<0.05$. 
Table 3. Plasma lipid and apolipoprotein levels (mean (SD)) by birth weight percentile and apo E genotype in girls

\begin{tabular}{|c|c|c|c|c|}
\hline & $\varepsilon 2 \varepsilon 3 * n=53$ & $\varepsilon 3 \varepsilon 3 n=292$ & $\varepsilon 3 \varepsilon 4 * n=66$ & All $n=411$ \\
\hline \multicolumn{5}{|l|}{ TC (mg/dl) } \\
\hline Low tertile & $164.3(18.3) \dagger$ & $186.5(28.9)$ & $194.2(29.7)$ & $186.0(29.6)$ \\
\hline Medium tertile & $169.5(21.3) \ddagger$ & $185.4(26.9)$ & $194.5(19.0)$ & $184.9(26.0)$ \\
\hline High tertile & $174.3(25.7) \dagger$ & $187.0(25.4)$ & $185.3(30.8)$ & $183.9(26.8)$ \\
\hline Whole group & $170.2(22.6) \dagger$ & $186.5(27.1)$ & $191.7(25.9)$ & $184.9(27.5)$ \\
\hline \multicolumn{5}{|l|}{ LDL-C (mg/dl) } \\
\hline Low tertile & $83.5(18.2) \dagger$ & $115.2(26.4)$ & $124.4(28.2)$ & $113.4(28.6)$ \\
\hline Medium tertile & $95.3(19.4) \dagger$ & $114.5(25.7)$ & $123.5(17.6)$ & $112.9(26.1)$ \\
\hline High tertile & $95.1(29.2) \ddagger$ & $113.6(23.9)$ & $115.7(26.5)$ & $110.6(26.0)$ \\
\hline Whole group & $92.3(24.1) \dagger$ & $114.3(25.4)$ & $121.2(23.4) \$$ & $112.3(26.9)$ \\
\hline \multicolumn{5}{|l|}{ Apo B (mg/dl) } \\
\hline Low tertile & $53.6(8.2) \dagger$ & $72.5(14.3)$ & $79.8(15.5)$ & $71.4(16.5)$ \\
\hline Medium tertile & $56.2(10.4) \dagger$ & 71.7 (13.9) & $78.3(9.1) \ddagger$ & $70.4(14.2)$ \\
\hline High tertile & $60.8(10.2) \dagger$ & $72.4(12.5)$ & $74.6(12.7)$ & $70.7(12.8)$ \\
\hline Whole group & $57.5(10.1) \dagger$ & $72.1(13.5)$ & $77.6(12.0) \dagger$ & $70.8(14.6)$ \\
\hline \multicolumn{5}{|l|}{$\mathrm{TG}(\mathrm{mg} / \mathrm{dl})$} \\
\hline Low tertile & $65.9(25.1)$ & $73.2(19.6)$ & $82.5(26.8)$ & $75.1(24.7)$ \\
\hline Medium tertile & $75.0(31.5)$ & $72.4(20.5)$ & $75.5(21.6)$ & $75.2(24.1)$ \\
\hline High tertile & $72.2(23.4)$ & $74.6(23.0)$ & $74.4(20.2)$ & 74.7 (22.9) \\
\hline Whole group & $71.4(22.4)$ & $73.8(21.4)$ & $76.5(22.4)$ & $75.0(23.8)$ \\
\hline \multicolumn{5}{|l|}{ HDL-C (mg/dl) } \\
\hline Low tertile & $67.2(16.5) \ddagger$ & $56.3(14.5)$ & $53.2(16.9)$ & $57.3(15.2)$ \\
\hline Medium tertile & $58.2(9.7)$ & $55.6(11.7)$ & $55.6(10.6)$ & $56.6(11.8)$ \\
\hline High tertile & $64.4(18.9)$ & $58.1(12.4)$ & $54.5(10.2)$ & $58.1(13.7)$ \\
\hline Whole group & $63.3(16.1) \dagger$ & $57.0(12.8)$ & $55.0(12.0)$ & $57.4(13.7)$ \\
\hline \multicolumn{5}{|l|}{ apo $A-I(\mathrm{mg} / \mathrm{dl})$} \\
\hline Low tertile & $142.1(18.8)$ & $134.3(17.5)$ & $127.9(23.0)$ & $135.3(18.6)$ \\
\hline Medium tertile & $137.8(14.8)$ & $133.8(18.2)$ & $134.9(14.9)$ & $134.4(17.5)$ \\
\hline High tertile & $145.6(18.2)$ & $138.8(17.4)$ & $131.5(17.6)$ & $138.3(18.0)$ \\
\hline Whole group & $142.4(17.4) \dagger$ & $135.9(17.7)$ & $132.2(17.9)$ & $136.0(18.1)$ \\
\hline
\end{tabular}

* Tukey test: $p$-values for differences in lipid levels between the $\varepsilon 3 \varepsilon 2$ or $\varepsilon 3 \varepsilon 4$ genotypes respectively, and the $\varepsilon 3 \varepsilon 3$ genotype.

$\dagger p<0.01$.

$\ddagger p<0.05$.

also measured in the RA 1000 after precipitation of apo B-containing lipoproteins with phosphotungstic acid and $\mathrm{Mg}$ (Boehringer Mannheim). LDL-C was calculated according to Friedenwald's formula. Plasma apo A-I and apo B concentrations were quantified by immunonephelometry (Array System, Beckman Instruments).

Apo E genotyping. Genomic DNA was prepared from leukocytes. For apo E genotyping, DNA was amplified by PCR using the primers 5'CGGGCACGGCTGTCCAAGGAG3' and 5'CAGCGCGCCCTGTTCCACGAG3' as described (15). The 244bp amplified fragment was restricted with the enzyme $H h a \mathrm{I}$, and the resulting DNA fragments were separated by an 8\% PAGE. Apo E genotype was determined by comparison with the combination of fragment sizes described by Hixon and Vernier (16).

Statistical analysis. Differences in mean values of anthropometric variables and lipid traits between boys and girls were tested by Student's $t$ test. Analysis of variance (ANOVA) was used to compare lipid and apolipoprotein levels and anthropometric measurement across genotypes in the whole sample, and by tertiles of birth weight, for boys and girls separately. When statistically significant differences arose $(p<0.05)$, differences between each pair of groups were assessed by the Tukey test.

Data were also analyzed using linear regression analysis. The linear regression analysis was performed with the lipid parameters as dependent variables and birth weight (in a continuous scale), two dummy variables for $\epsilon 2$ and $\epsilon 4$ alleles and the interactions between those variables as independent variables. This type of analysis was aimed to examine the independent relationship of the lipid variables with birth weight, apo E genotype and their interactions.

Statistical analyses were performed using the SPSS software package, version 9.0.

\section{RESULTS}

Descriptive statistics for the age, weight, height, BMI, birth weight, and plasma lipid levels by gender are given in Table 1 . The average birth weight of girls was lower than for boys. In addition, girls showed significantly lower apo A-I and higher apo B plasma levels than boys.

Due to the typically small representation of the $\epsilon 2 \epsilon 2, \epsilon 2 \epsilon 4$, and $\epsilon 4 \epsilon 4$ genotypes, we focused our analyses on subjects with the three most common genotypes: $\epsilon 2 \epsilon 3, \epsilon 3 \epsilon 3$, and $\epsilon 3 \epsilon 4$. The distribution of the apo $E$ genotypes did not differ between girls and boys, and was similar to that reported in South European countries $(17,18)$. Mean values for anthropometric variables were similar across apo E genotypes (data not shown).

In both sexes, mean concentrations of plasma TC, LDL-C and apo B varied significantly $(p<0.01)$ across the apo E genotypes. Boys and girls with the $\epsilon 2 \epsilon 3$ genotype had significantly $(p<0.01)$ lower plasma TC, LDL-C, and apo B 
concentrations than those found in the $\epsilon 3 \epsilon 3$ genotype. On the other hand, children with the $\epsilon 3 \in 4$ genotype tended to have higher values for these parameters than children with the $\epsilon 3 \epsilon 3$ genotype (Tables 2 and 3 ).

However, the effect of apo E genotype on lipid levels substantially varied across tertiles of birth weight. The frequencies of apo E polymorphisms did not differ between children of different birth weights (data not shown). To evaluate the effect of birth weight, plasma lipid and lipoprotein levels in the three tertiles of birth weight were calculated for each apo E genotype in boys (Table 2) and girls (Table 3). Considering all apo E genotypes together, those with low birth weight tended to have elevated TC, LDL-C, apo B and TG, and diminished apo A-I plasma concentrations, though the trends were not statistically significant.

As shown in Table 2, using the $\epsilon 3$ allele homozygosity as reference, the overall lowering effect of the $\epsilon 2$ allele in boys in the lowest birth weight tertile was much higher $(10.5 \%$ on TC $(p<0.01), 20.2 \%$ on LDL-C $(p<0.01)$ and $18.8 \%$ on apo B $(p<0.01))$ than in those in the highest birth weight tertile, where the effect of the $\epsilon 2$ allele was $5.6 \%$ on TC levels, $10.3 \%$ on LDL-C levels and reached statistical significance only for apo B levels $(12.6 \%, p<0.01)$. Similar effects of $\epsilon 2$ allele on TC, LDL-C and apo B between the low and the high tertiles of birth weight were observed in girls (Table 3 ). The lowering effect of the $\epsilon 2$ allele was $12 \%(p<0.01)$ on TC, $27.5 \%(p<$ $0.01)$ on LDL-C and $26 \%(p<0.01)$ on apo B levels in the lowest tertile group of girls, and $7 \%(p<0.05)$ on TC, $16 \%(p$ $<0.05)$ on LDL-C and $16 \%(p<0.01)$ on apo B levels in the highest tertile of birth weight. In girls, the elevating effect of the $\epsilon 2$ allele on HDL-C was statistically significant in the low tertile but not in the high tertile. Figure 1 shows that, for both sexes, the decrease in TC, LDL-C and apo B levels found in children of the $\epsilon 2 \epsilon 3$ as compared with children of the $\epsilon 3 \epsilon 3$ genotype is lower as the body weight increases.

Table 4 presents the results of the linear regression analysis (regression coefficients, standard errors, $p$ values, and the value of $\mathrm{R}^{2}$ for the model). For both sexes, the analysis shows that $\epsilon 2$ was associated with a statistically significant decrease in plasma TC, LDL-C, and apo B. In addition, it demonstrates a positive and significant interaction between birth weight and $\epsilon 2$, which may explain the fact that the decrease in TC, LDL-C, and apo B associated with the $\epsilon 2$ allele is more marked the lower the birth weight. However, birth weight and $\epsilon 4$ variables or their interactions did not show an independent relationship with none of the lipid parameters studied.

\section{DISCUSSION}

We have examined the possible interactions between apo E genotypes and anthropometric variables in our sample of 491 boys and 442 girls. The analysis of the apo E genotypes and plasma lipid levels in these prepubertal children revealed the expected effect of the $\epsilon 2$ and $\epsilon 4$ alleles on plasma TC, LDL-C and apo B levels; $\epsilon 2$ decreases whereas $\epsilon 4$ increases these lipid values. Interestingly, we noted a greater effect of the apo $\mathrm{E}$ polymorphism on TC, LDL-C and particularly the apo B levels in children with birth weight in the low tertile than those in the
A) Total cholesterol (mg/dl)

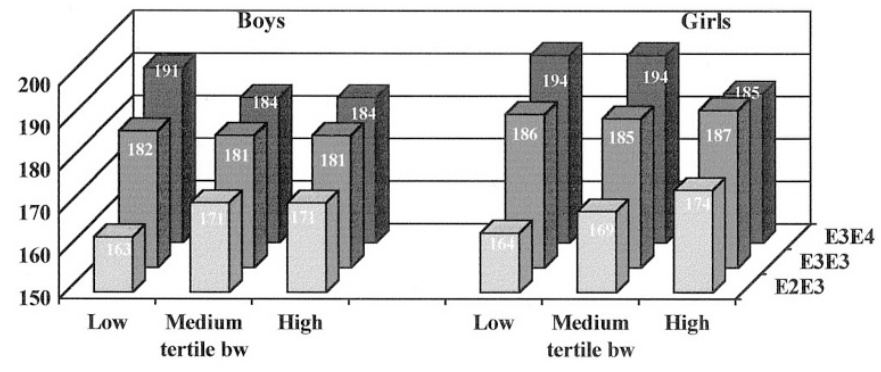

B) LDL-cholesterol (mg/dl)

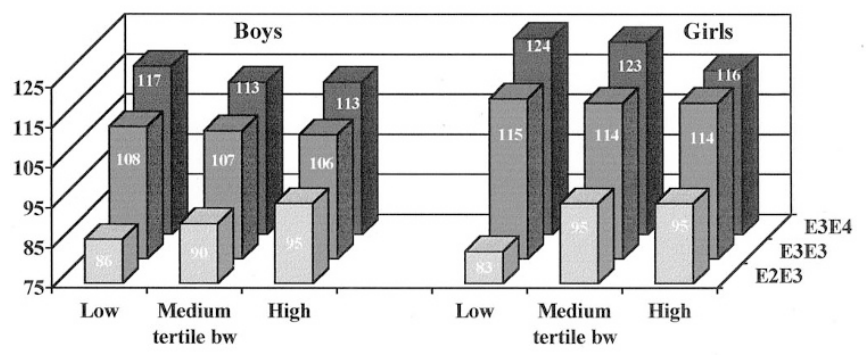

C) Apo B (mg/dl)

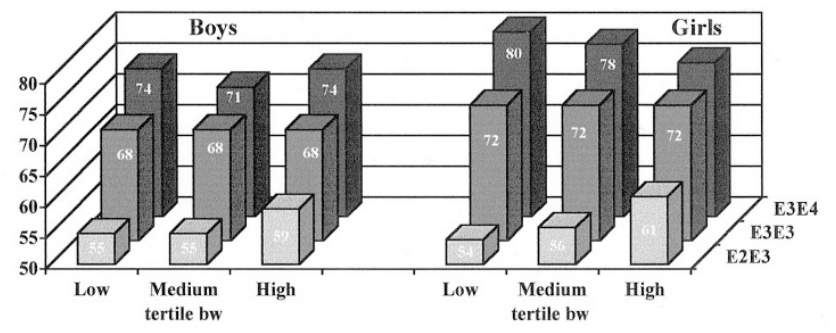

Figure 1. Lipid measurements in boys and girls by birth weight and apo $\mathrm{E}$ genotype. (A) Total cholesterol. (B) LDL-cholesterol. (C) Apo B levels. In boys, low tertile $=$ birth weight $<3.1 \mathrm{~kg}$; medium tertile between $3.1 \mathrm{~kg}$ and $3.8 \mathrm{~kg}$; high tertile $=$ birth weight $>3.8 \mathrm{~kg}$. In girls, low tertile $=$ birth weight $<2.9 \mathrm{~kg}$; medium tertile between $2.9 \mathrm{~kg}$ and $3.5 \mathrm{~kg}$; high tertile $=$ birth weight $>3.5 \mathrm{~kg}$.

high tertile. Compared with the $\epsilon 3 \in 3$ subjects, the reducing effect of the $\epsilon 2$ allele and the elevating effect of the $\epsilon 4$ allele on lipid levels was greater in those children with the lowest birth weight. The major differences were mainly associated with effects of the $\epsilon 2$ allele.

As the apo E allelic frequencies did not differ in boys and girls with different birth weights, the association of a particular allele with decreased or increased levels of the traits is not the explanation for those findings. Thus, a different allele seems to impact TC, LDL-C, and apo B levels, depending on birth weight.

It is accepted that variations in plasma lipid levels are determined, in part, by polymorphisms in the apo E gene (7-13) and also by birth or infancy weight (19). However, the interaction of anthropometric variables with the specific effects of apo E genotype on those variables remains unclear.

Henry et al. (20) reported that the LDL lowering effect of the $\epsilon 2$ allele and the raising effect of the $\epsilon 4$ allele was greater in a 
Table 4. Results of the regression analysis of lipid variables on birthweight $(B W)$, apo E genotype and their interactions, by sex

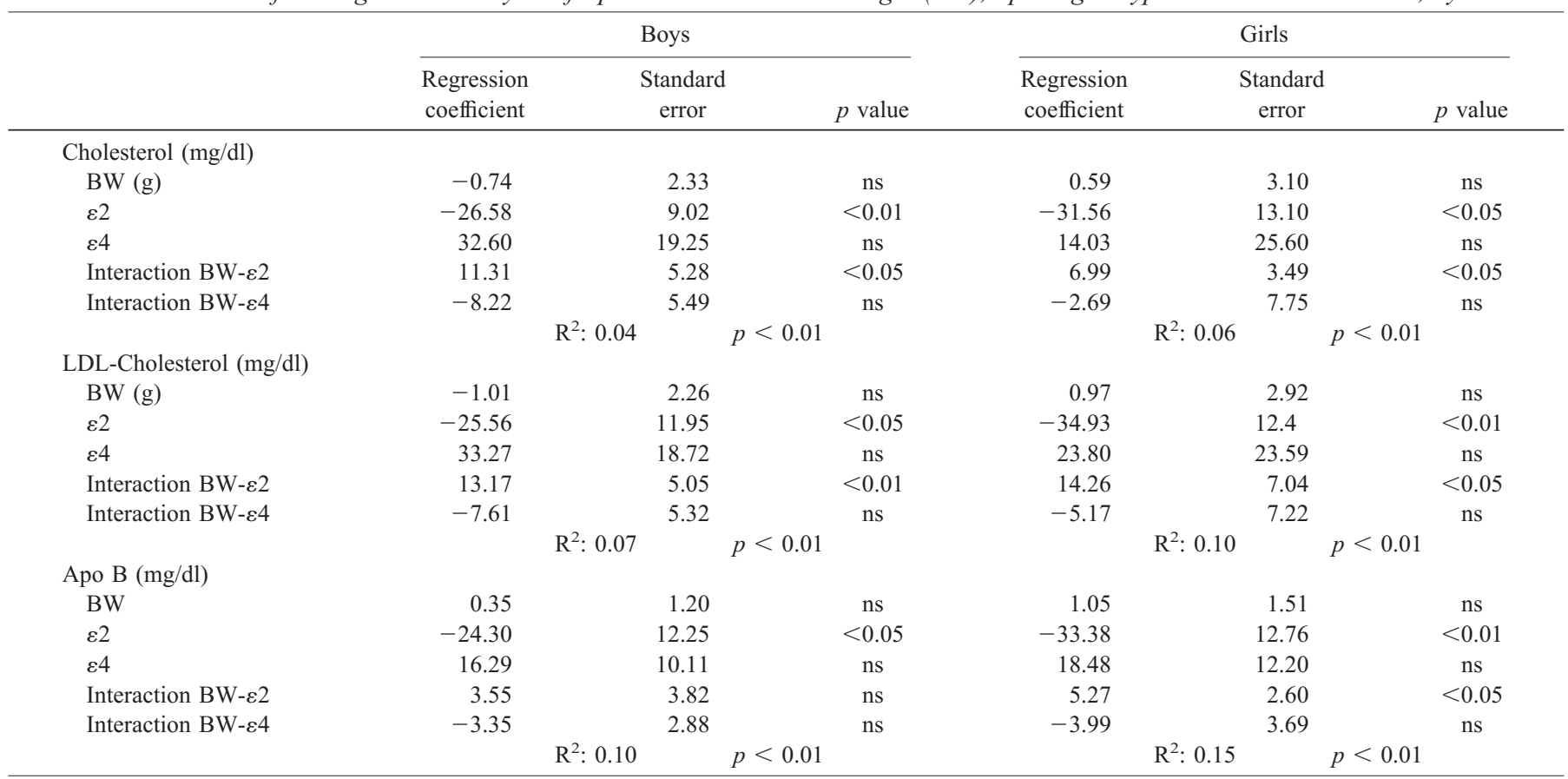

group of adults with low infant weight as compared with a group with high infant weight, although in their study this effect did not reach statistical significance. Moreover, as a mechanistic explanation for these observations, the authors suggested that changes in the apo E gene expression had been programmed by in utero nutritional events.

The large number of children included in our study provided a sample of sufficient size to compare the effect of apo E genotype on lipid levels according to birth weight. Furthermore, our study focused on children of specific prepubertal age (average age of $6.7 \mathrm{y}$ ), allowing us to study the effects of the gene without complications of sex hormones. Moreover, these children were studied at an age where environmental or lifestyle factors typical of adult age (stress, alcohol, tobacco, etc.) are not present and thus, do not complicate the effect of apo E genotype on lipids.

The major function of the apo E ligand is regulate the hepatic clearance of $\mathrm{TG}$ rich lipoproteins of intestinal and hepatic origin via the apo $\mathrm{B}$ and apo E LDL receptor and the LDL receptor-related protein (21). The Apo E2 isoform has a lower affinity to apo E receptors than E3 and E4 isoforms (22). The differences in cholesterol absorption and postprandial remnant clearance between phenotypes, due to the different isoforms, may lead to up-regulation of hepatic LDL-receptors in subjects with E2-isoform and a lowering of serum cholesterol levels. Conversely, efficient uptake of apo E4-containing triglyceride-rich particles causes hepatic lipid accumulation with down-regulation of LDL-receptors and an increase in serum cholesterol levels.

Elevated serum cholesterol concentrations in adult life are associated with impaired growth during late gestation, when fetal under-nutrition exerts a disproportionate effect on liver growth. Impaired liver growth may permanently alter LDL-C metabolism (19). If reduced fetal growth leads to poor liver growth, it may also cause a down-regulation of hepatic receptors. Consequently, the different properties of each isoform in determining differences in lipid metabolism would be intensified by the hepatic programming of the receptor gene expression in the case of apo E2 and apo E4 isoforms and neutral in the case of apo E3.

Our results have allowed us to confirm that the effects of $\epsilon 2$ and $\epsilon 4$ alleles on apo B-containing lipoproteins in this schoolbased survey were as expected. More importantly, our observations demonstrate that the extent of the lipid increasing or decreasing effects associated with an allele were modulated by birth weights, being more pronounced in subjects of low birth weight. Thus, in children with the $\epsilon 2$ allele, a birth weight in the lowest tertile determines a much-less atherogenic profile at the prepubertal age than a high birth weight. Taking into account the prevalence of these alleles and that the apo $\mathrm{E}$ polymorphism appears to be the main genetic regulator of plasma lipids in our population, the interaction of apo $E$ genotype and birth weight may represent a critical determinant of TC, LDL-C, and apo B levels, and, therefore, of atherosclerosis.

Acknowledgments. We thank Miguel Martín and Sara Guerras for technical assistance.

\section{REFERENCES}

1. Barker DJP, Winter PD, Osmond C, Margetts B, Simmonds SJ 1989 Weight in infancy and death from ischaemic heart disease. Lancet 2:577-580

2. Fall CH, Vijayakumar M, Barker DJ, Osmond C, Duggleby 1995 Weight in infancy and prevalence of coronary heart disease in adult life. BMJ 310:17-19

3. Mahley RW, Huang Y 1999 Apolipoprotein E: from atherosclerosis to Alzheimer's disease and beyond. Curr Opin Lipidol 10:207-217

4. Gerdes LU, Klausen IC, Sihm I, Faergeman O 1992 Apolipoprotein E polymorphism in a Danish population compared to findings in 45 other study populations around the world. Genet Epidemiol 9:155-167 
5. de Knijff P, Havekes LM 1996 Apolipoprotein E as a risk factor for coronary heart disease: a genetic and molecular biology approach. Curr Opin Lipidol 7:59-63

6. Wilson PW, Schaefer EJ, Larson MG, Ordovás JM 1996 Apolipoprotein E alleles and risk of coronary disease. A meta-analysis. Arterioscler Thromb Vasc Biol 16:1250 1255

7. Sing CF, Davignon J 1985 Role of the apolipoprotein E polymorphism in determining normal plasma lipid and lipoprotein variation. Am J Hum Genet 37:268-285

8. Kamboh MI, Aston CE, Ferrell RE, Hamman RF 1993 Impact of apolipoprotein E polymorphism in determining interindividual variation in total cholesterol and low density lipoprotein cholesterol in Hispanics and non-Hispanic whites. Atherosclerosis 98:201-211

9. Gómez-Coronado D, Alvarez JJ, Entrala A, Olmos JM, Herrera E, Lasuncion MA 1999 Apolipoprotein E polymorphism in men and women from a Spanish population: allele frequencies and influence on plasma lipids and apolipoproteins. Atherosclerosis 147:167-176

10. Xu CF, Talmud PJ, Angelico F, Del Ben M, Savill J, Humphries SE 1991 Apolipoprotein E polymorphism and plasma lipid, lipoprotein, and apolipoprotein levels in Italian children. Genet Epidemiol 8:389-398

11. Lehtimaki T, Porkka K, Viikari J, Ehnholm C, Akerblom HK, Nikkari T 1994 Apolipoprotein $\mathrm{E}$ phenotypes and serum lipids in newborns and 3-year-old children: the Cardiovascular Risk in Young Finns Study. Pediatrics 94:489-493

12. Herrmann W, Hanf S, Kaffarnik H, Motzny S, Reissner J, Steinmetz A 1994 The influence of apolipoprotein E polymorphism on plasma concentrations of apolipoprotein B and A-I during the first year of life. Pediatrics 93:296-302

13. Lapinleimu H, Vukari J, Nunikoski H, Tuominen J, Ronnemaa T, Valimaki I, Marniemi J, Jokinen E, Ehnholm C, Simell O 1997 Impact of gender, apolipoprotein
E phenotypes, and diet on serum lipids and lipoproteins in infancy. J Pediatr 131:825-832

14. Rodriguez Artalejo F, Garcés C, Lasunción MA, Martin Moreno JM, Gorgojo L y de Oya M 1999 Estudio cuatro provincias: principales objetivos y diseño. Rev Esp Cardiol 52:319-326

15. Tsai MS, Tangalos EG, Petersen RC, Smith GE, Schaid DJ, Kokmen E, Ivnik RJ, Thibodeau SN 1994 Apolipoprotein E: Risk Factor for Alzheimer disease. Am J Hum Genet 54:643-649

16. Hixson JB, and Vernier DT 1990 Restriction isotyping of human Apolipoprotein E by gene amplification and cleavage with Hhal. J Lipid Res 31:545-548

17. James RW, Boemi M, Giansanti R, Fumelli P, Pometta D 1993 Underexpression of the apolipoprotein E4 isoform in an Italian population. Arterioscler Thromb 13:14561249

18. Muros M, Rodríguez-Ferrer C 1996 Apolipoprotein E polymorphism influence on lipids, apolipoproteins and $\mathrm{Lp}(\mathrm{a})$ in a Spanish population underexpressing apo E4. Atherosclerosis 121:13-21

19. Barker DJ, Martyn CN, Osmond C, Hales CN, Fall CHD 1993 Growth in utero and serum cholesterol concentrations in adult life. BMJ 307:1524-1527

20. Henry JA, Bolla M, Osmond C, Fall C, Barker DJ, Humphries SE 1997 The effects of genotype and infant weight on adult plasma levels of fibrinogen, factor VII, and LDL cholesterol are additive. J Med Genet 34:553-558

21. Beisiegel U, Weber W, Ihrke G, Herz J, Stanley KK 1989 The LDL-receptor-related protein, LRP, is an apolipoprotein E-binding protein. Nature 341:162-164

22. Weisgraber KH, Innerarity TL, Mahley RW 1982 Abnormal lipoprotein receptorbinding activity of the human $\mathrm{E}$ apoprotein due to cysteine-arginine interchange at a single site. J Biol Chem 257:2518-2521 